\title{
Numerical Evaluation of an Ejector-Enhanced Resonant Pulse Combustor with a Poppet Inlet Valve and a Converging Exhaust Nozzle
}

\author{
Shaye Yungster* \\ Ohio Aerospace Institute, Cleveland, OH 44142 \\ Daniel E. Paxson ${ }^{\dagger}$ and Hugh D. Perkins \\ NASA Glenn Research Center, Cleveland, OH 44135
}

\begin{abstract}
A computational investigation of a pressure-gain combustor system for gas turbine applications is presented. The system consists of a valved pulse combustor and an ejector, housed within a shroud. The study focuses on two enhancements to previous models, related to the valve and ejector components. First, a new poppet inlet valve system is investigated, replacing the previously used reed valve configuration. Secondly, a new computational approach to approximating the effects of choked turbine inlet guide vanes present immediately downstream of the Ejector-Enhanced Resonant Pulse Combustor (EERPC) is investigated. Instead of specifying a back pressure at the EERPC exit boundary (as was done in previous studies) the new model adds a converging-diverging $(\mathrm{CD})$ nozzle at the exit of the EERPC. The throat area of the CD nozzle can be adjusted to obtain the desired back pressure level and total mass flow rate. The results presented indicate that the new poppet valve configuration performs nearly as well as the original reed valve system, and that the addition of the CD nozzle is an effective method to approximate the exit boundary effects of a turbine present downstream of the EERPC. Furthermore, it is shown that the more acoustically reflective boundary imposed by a nozzle as compared to a constant pressure surface does not significantly affect operation or performance.
\end{abstract}

\section{Nomenclature}

$$
\begin{array}{ll}
D_{t h}^{C D} & =\text { CD nozzle throat diameter (Fig. 10) } \\
D_{t h}^{E J} & =\text { ejector throat diameter (Fig. 9) } \\
E E R P C & =\text { ejector-enhanced resonant pulse combustor } \\
\dot{m}_{p r i} & =\text { primary massflow rate } \\
\dot{m}_{\text {sec }} & =\text { secondary massflow rate } \\
\dot{m}_{t o t} & =\text { total massflow rate } \\
p & =\text { pressure } \\
p_{b} & =\text { back pressure } \\
p_{0} & =\text { inlet total pressure } \\
R P C & =\text { resonant pulse combustor } \\
T & =\text { temperature } \\
T_{0} & =\text { inlet total temperature } \\
t & =\text { time } \\
U & =\text { axial velocity }
\end{array}
$$

\footnotetext{
* Senior Scientist, Senior AIAA Member.

${ }^{\dagger}$ Aerospace Engineer, Associate Fellow AIAA.

¥ Aerospace Engineer, AIAA Member.
} 


$\begin{array}{ll}x & =\text { axial coordinate }(\text { Fig. } 5) \\ \boldsymbol{\beta} & =\text { bypass ratio }\left(\dot{m}_{\text {sec }} / \dot{m}_{p r i}\right) \\ \phi & =\text { equivalence ratio } \\ \boldsymbol{\xi}, \boldsymbol{\eta} & =\text { generalized coordinates } \\ \sigma & =\text { standard deviation }\end{array}$

\section{Introduction}

Gas turbine engine combustors based on steady, constant pressure combustion actually incur total pressure losses (typically ranging from 4 to 8 percent ${ }^{1}$ ) due to thermodynamic losses associated with heat release at finite Mach number (i.e. Rayleigh losses), and due to aerodynamic losses associated with the intricate flow path. This total pressure loss translates into a reduction in thermal efficiency.

The benefits of eliminating this loss and achieving some level of pressure gain have been investigated by Jones and Paxson. ${ }^{2}$ Their analysis indicated that even a modest pressure rise across the combustor can result in substantial improvement in specific fuel consumption. The potential benefits of pressure-gain combustion have motivated a large number of experimental and analytical investigations of various concepts that include detonation based devices, wave rotors and resonant pulse combustors (RPC), all of which are unsteady devices.

High pressure gain is not the sole determinant of improved performance in the gas turbine environment. Several other factors need to be considered also, such as mechanical complexity, flow non-uniformities, mechanical and thermal loading of pressure gain devices and emission of pollutants. In light of these considerations, the RPC concept, when used in combination with ejector systems, has several advantages over alternative pressure gain combustion concepts, and is the subject of the present study.

Resonant pulse combustors are unsteady, thermo-acoustic devices in which heat released by combustion is coupled with the acoustic waves. They are relatively simple devices, and thereby avoid the mechanical complexities of higher pressure-gain concepts such as wave rotors and detonation based devices. Flow non-uniformities at the exit of ejector-enhanced pulse combustor devices have been shown to be substantially lower in comparison to those observed in detonation based devices. ${ }^{3}$ This smoothing aspect of ejector-enhanced pulse combustor based systems is critical for maintaining high turbomachinery performance. In addition, the emissions of oxides of nitrogen in RPCs are potentially lower than in conventional combustor systems. ${ }^{4}$ The main disadvantage of RPC based systems is that the pressure gain attainable is typically lower than that for wave rotors or detonation based devices. On the other hand, the lower pressure-gain carries with it the implication of lower thermal and mechanical stresses. As such, the theoretical disadvantage may be a practical advantage.

Aerospace applications of pressure-gain combustion systems necessitates operation at high pressure conditions. Preliminary calculations of Ejector-Enhanced Resonant Pulse Combustor (EERPC) configurations operating at high pressure conditions $(10 \mathrm{bar})$ produced pressure gains significantly lower than those observed experimentally and computationally at atmospheric conditions. ${ }^{5} \mathrm{~A}$ subsequent study ${ }^{6}$ identified the factors limiting the pressure-gain at high pressure conditions and investigated the effects of fuel injection and air mixing characteristics on performance. New pulse combustor configurations were developed in Ref. 6 which were able to achieve performance levels at high pressure conditions comparable to those observed at atmospheric conditions.

The study presented in Ref. 6 only considered the pulse combustor by itself, which is not suitable to replace a conventional combustor in a gas turbine engine since, among other factors, the minimum fuel-to-air ratio for successful RPC operation produces an effluent which is too hot for downstream turbomachinery. As such, some amount of bypass air is required.

In recent studies, 7,8 the performance of various EERPC configurations operating at high pressure conditions were investigated, focusing on the effects of equivalence ratio and ejector throat area. The philosophy behind this approach was to take advantage of the relatively efficient mixing and pumping characteristics of unsteady ejectors

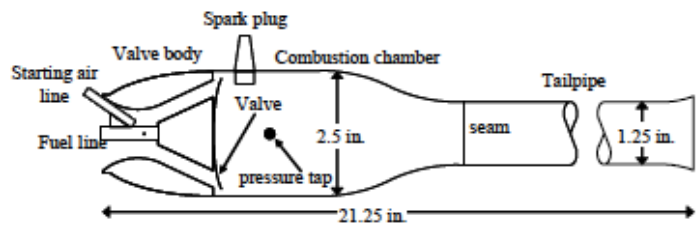

Figure 1. Schematic of resonant pulse combustor. 
for introducing the bypass flow. The results demonstrated pressure gains of nearly $3 \%$ at gas turbine compatible combustor temperature ratios, with nearly smooth exit flow, while maintaining a NOx emission index under ten.

Figure 1 shows a schematic of a RPC. The reed valve is the only moving part in the RPC. This valve is self-actuated, its position is determined at every instant by the pressure differential existing between the inlet and combustor sides. In the previous studies, ${ }^{5-8}$ a vertically sliding valve model (shown in Fig. 2), that approximated the reed valve action, was utilized.

In practice, reed valves can operate for only a limited number of cycles before they fail due to the high temperatures and impact forces experienced by them during operation, and also due to the fact that they have to be very thin and flexible in order to open and close quickly. Furthermore, the sliding valve model shown in Fig. 2 does not represent a mechanically practical motion (i.e. a symmetric disk that moves radially). Therefore, in the present study, a more feasible and robust poppet valve system is investigated.

\section{Computational Model of Poppet Valve}

Shown notionally in Fig. 3a, the poppet valve would be actuated (similar to those found in internal combustion engines) and possibly actively controlled, with a translating motion in the axial direction. Currently, this translation is difficult to implement in the CFD code used for this work. As such, the arrangement shown in Fig. $3 b$, which is relatively easy to implement and fluidically similar, is used in the present study. The approximate poppet valve model consists of a rotating valve that closes the airflow when it's in the vertical position, and opens up by rotating clockwise.

Figure 4 shows details of the generalized coordinate grid structure in the valve region. The grid is uniformly distributed in the $\xi$ direction. This grid distribution allows for a simple simulation of the opening and closing of the valve. In Fig. 4, the valve's left wall is specified at the $\xi_{n}$ grid line. The valve's right wall will then

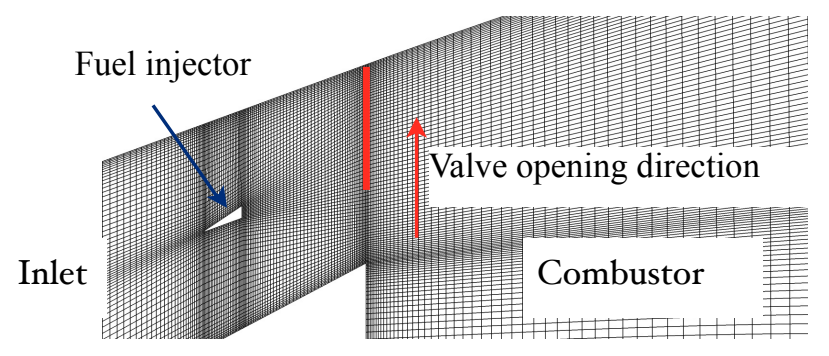

Figure 2. Vertically sliding valve model..$^{5-8}$
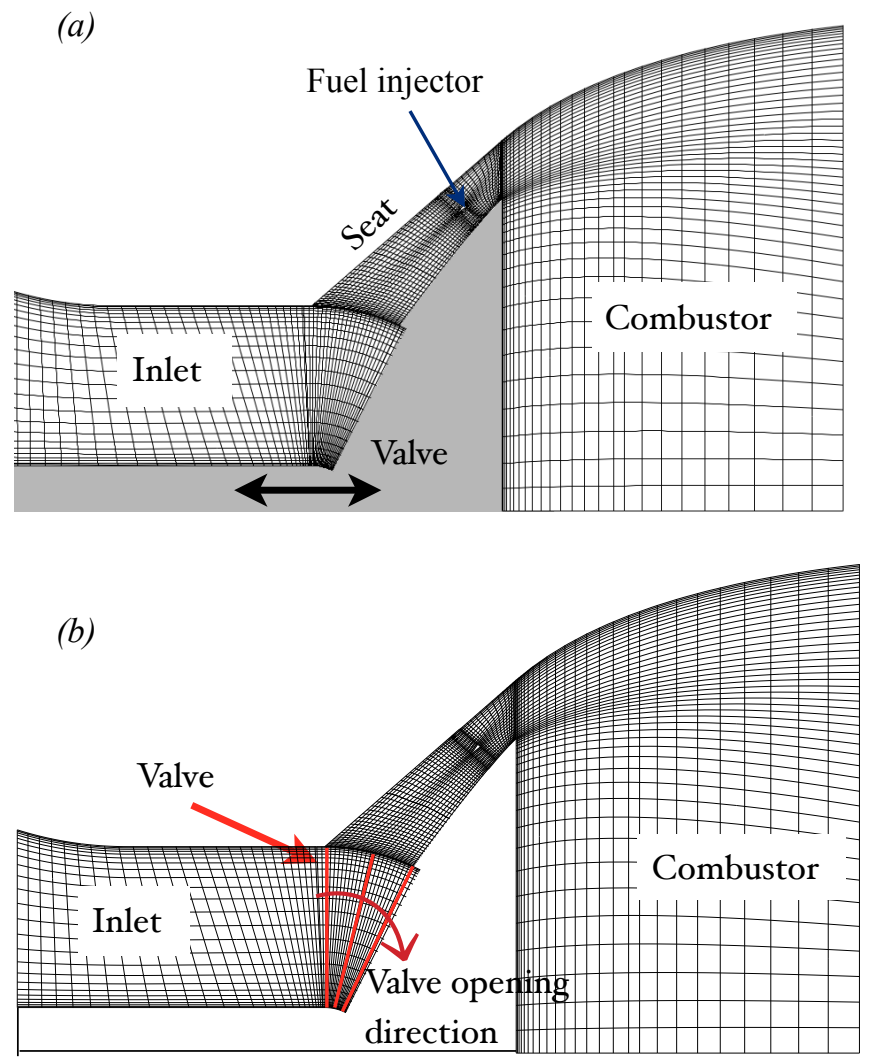

Figure 3. Poppet inlet valve models; (a) translating valve, (b) rotating valve model (only every fourth grid line is shown for clarity).

be located at the $\xi_{\mathrm{n}+1}$ grid line. Since the grid is uniformly distributed in the $\xi$ direction, opening of the valve is carried out by moving the left wall boundary definition of the valve from $\xi_{\mathrm{n}}$ to $\xi_{\mathrm{n}+1}$ and the right wall from $\xi_{\mathrm{n}+1}$ to $\xi_{\mathrm{n}}$ +2 . Closing of the valve is done similarly in the counter clockwise direction. The valve moves in discrete increments as its rotating motion reaches the neighboring $\xi$ gridline.

The valve wall boundary condition is implementing by specifying zero velocity in the $\eta$ direction $\left(v_{\eta}=0\right)$, and a velocity equal to $v_{\xi}=\omega r$, in the $\xi$ direction, where $\omega$ is the angular velocity of the valve and $r$ is the radial 
position of a given point on the valve surface. The angular velocity used in the calculations presented in this paper was $0.76 \mathrm{rad} / \mathrm{ms}$, corresponding to an opening time of $0.8 \mathrm{~ms}$. The pressure is obtained by solving the normal momentum equation at the wall.

This model is relatively simple to implement, although special care is needed to maintain accurate communication among all the connecting blocks for all valve positions. The main disadvantage of this approach is that the boundary layer on the valve surface is not well resolved.

An important parameter in this model is the maximum valve opening angle, $\theta_{\mathrm{v}}$ (see Fig. 4). This angle can affect the mass flow rate through the RPC. Initial calculations with $\theta_{\mathrm{v}}=25^{\circ}$ resulted in a low airflow rate $(0.16 \mathrm{~kg} / \mathrm{s})$ and poor performance. Increasing this angle to $35^{\circ}$ resulted in airflow of $0.22 \mathrm{~kg} / \mathrm{s}$, close to that obtained in the previous studies (refs 5-8). Further increase to $\theta_{\mathrm{v}}=45^{\circ}$ did not result in further changes in airflow through the RPC.

Opening and closing of the valve are specified such that opening occurs when the pressure at the head-end of the RPC falls below $p_{0}$, and closing closing occurs when the same pressure increases above $p_{0}$ later in the cycle.

The numerical simulations of the RPC are carried out using a Computational Fluid Dynamics (CFD) code developed in-house ${ }^{9,10}$. The analysis is based on the axisymmetric, unsteady, Reynolds-averaged

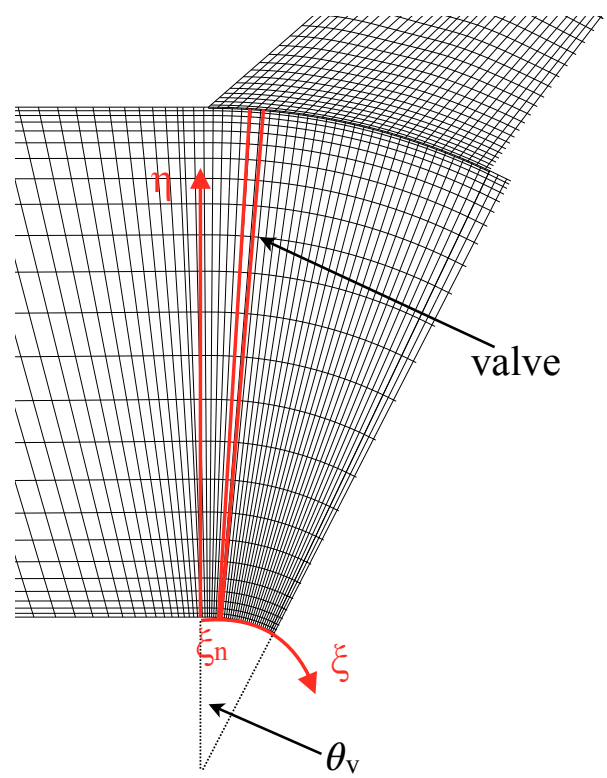

Figure 4. Grid distribution in the valve zone. Navier-Stokes equations for a multi-species, thermally perfect, chemically reacting gas. The turbulence model used in the calculations is the Spalart-Allmaras one-equation mode ${ }^{11}$. Adiabatic walls were assumed for all cases considered, and the grid had a minimum wall spacing of $1 \times 10^{-3}$ in (resulting in values for $\mathrm{y}^{+}$of order one). The numerical method used for solving the governing equations is described in detail in Refs. 9 and 10.

The fuel considered in this study is gaseous Jet- $\mathrm{A}$, which is modeled as a $\mathrm{C}_{11} \mathrm{H}_{21}$ species. A reduced combustion mechanism, based on the model developed by Ajmani et al. ${ }^{12}$, consisting of 10 elementary reactions among 11 reacting species was utilized. The complete reaction mechanism is given in Refs. 5 and 6.

\section{Numerical Simulations}

The present study focuses on two enhancements to previous computational models, related to the valve and ejector components. First, the new poppet inlet valve model is investigated for a RPC configuration without an ejector (section IIIA). Subsequently, the new computational approach to approximating the effects of choked turbine inlet guide vanes, present immediately downstream of the EERPC, is investigated in section IIIB.

All the numerical simulations presented here were run for multiple cycles until the overall flowfield approached periodicity (limit cycle). This typically required 8-12 cycles for the RPC configurations without an ejector, and up to 25 cycles for EERPC configurations. A typical EERPC case having 170000 grid points required 16 hours of walltime per cycle using 24 processors on the NAS supercomputer "Endeavour".

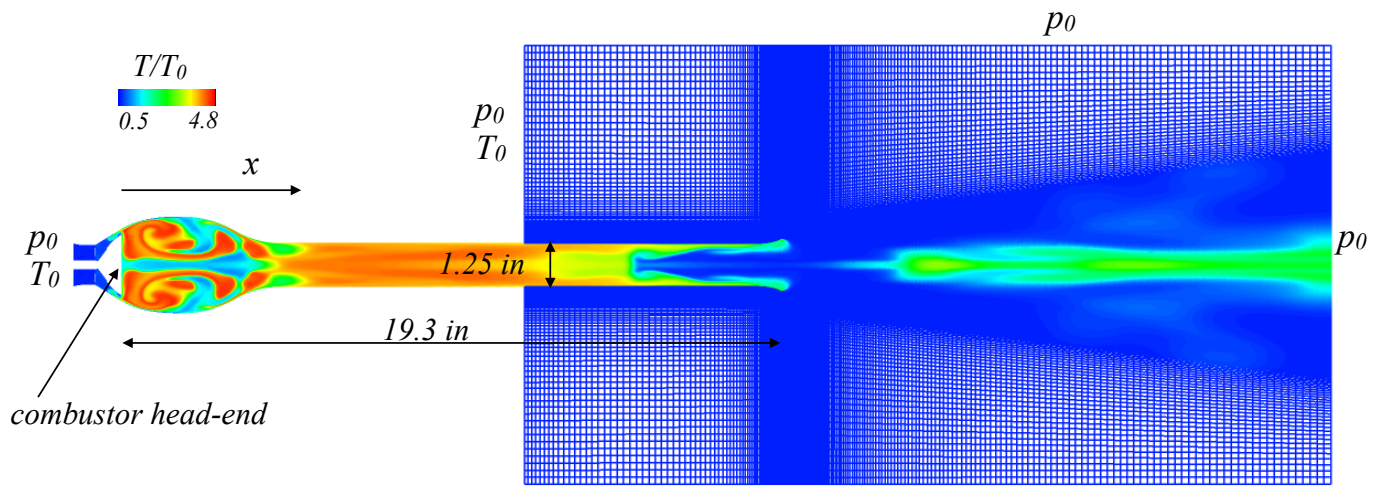

Figure 5. RPC computational domain, and temperature contours at start of cycle. 


\section{A. Simulations of RPCs with a Poppet Inlet Valve}

Figure 5 shows the computational domain used for the RPC calculations (without the ejector), and also shows temperature contours at the start of the cycle. A specified total pressure and total temperature boundary condition is applied at the inlet entrance. All the cases presented in this paper consider inflow conditions of $p_{0}=10 \mathrm{bar}$ and $T_{0}=$ $550 \mathrm{~K}$, which may be considered as representative conditions for the exit of a modern turbofan compressor at cruise conditions $^{6}$. The other boundaries (Fig. 5) are treated as either subsonic inflow, where the total pressure $\left(p_{0}\right)$ and total temperature $\left(T_{0}\right)$ are specified, or subsonic outflow, where the static pressure is imposed. In this study, the static pressure was set equal to the total pressure $p_{0}$.

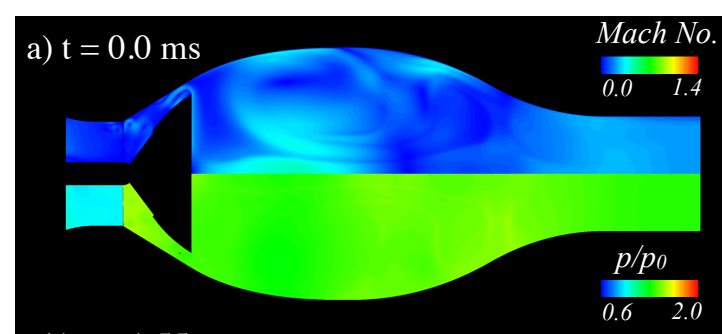

b) $\mathrm{t}=1.55 \mathrm{~ms}$

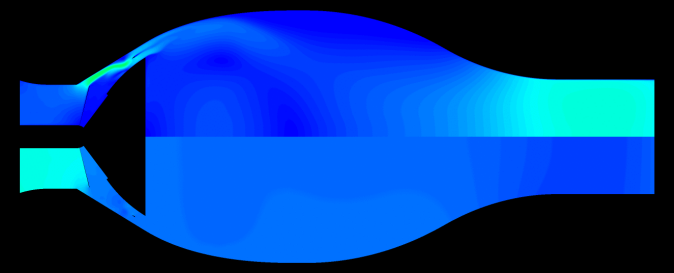

c) $\mathrm{t}=1.70 \mathrm{~ms}$

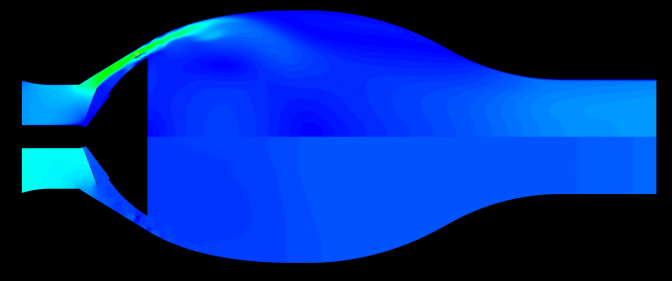

d) $\mathrm{t}=1.85 \mathrm{~ms}$

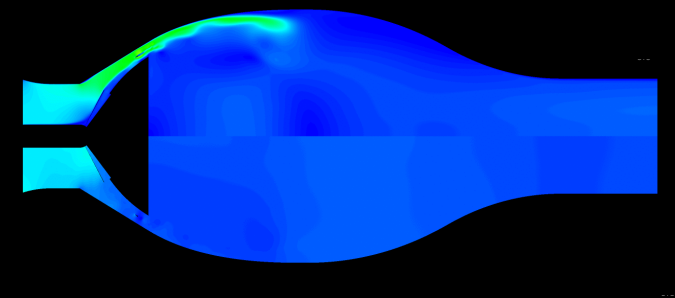

e) $\mathrm{t}=2.00 \mathrm{~ms}$

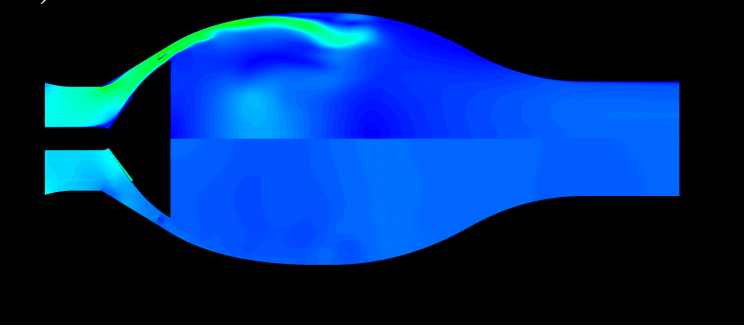

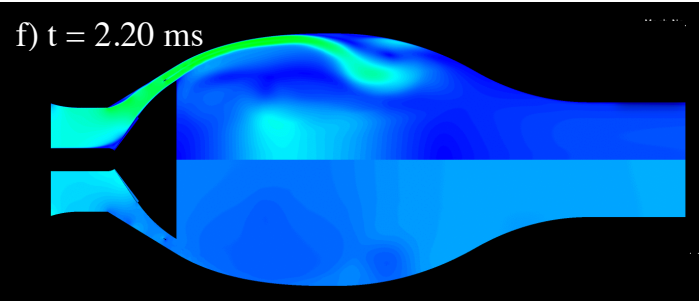

g) $\mathrm{t}=2.40 \mathrm{~ms}$

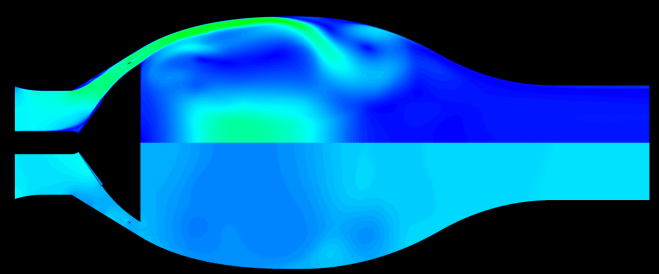

h) $\mathrm{t}=2.60 \mathrm{~ms}$

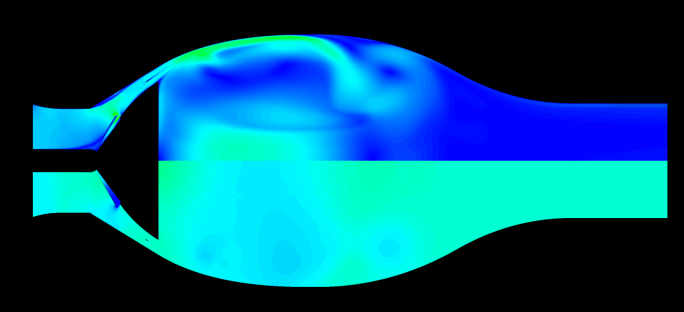

i) $\mathrm{t}=2.80 \mathrm{~ms}$

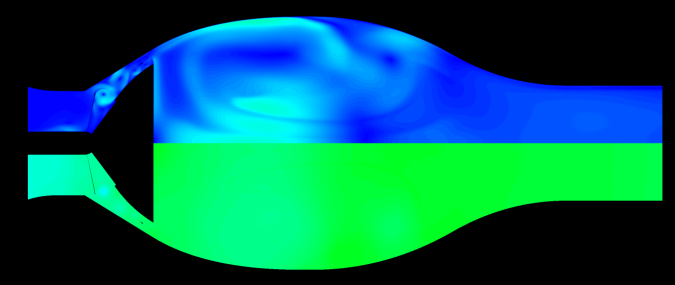

j) $\mathrm{t}=2.95 \mathrm{~ms}$

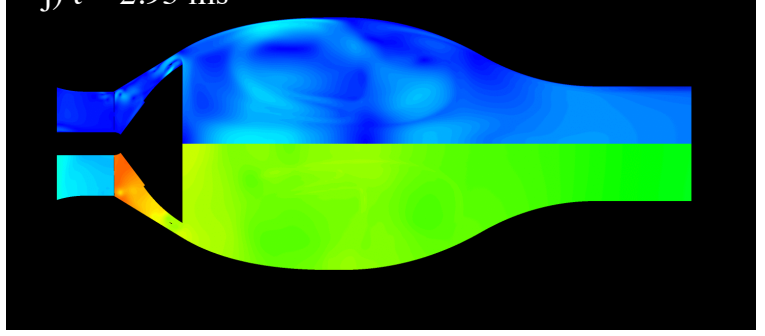

Figure 6. Mach number (top half) and pressure (bottom half) contours during one RPC cycle.

American Institute of Aeronautics and Astronautics 
The flow and combustion processes during one cycle of the RPC operation are presented in figs 6 and 7. Figure 6 shows Mach number contours (top half) and pressure ratio contours (bottom half) during one cycle. Only part of the RPC is shown for clarity. This figure shows the details of the valve operation. Figure 6a shows the flowfield just after the valve has fully closed (vertical position). The valve begins to open at $\mathrm{t}=1.30 \mathrm{~ms}$, just as the pressure at the head-end of the combustor drops below $p_{0}$, and air is seen entering the combustor in figs. 6b-6d. The position of the valve in these figures can be identified by the jump in pressure and Mach number across the valve. The valve is fully open at $\mathrm{t}=2.03 \mathrm{~ms}$ (Fig.6e). The valve remains open until the pressure at the head-end of the combustor increases above $p_{0}$. This occurred at $\mathrm{t}=2.59 \mathrm{~ms}$, at which point the valve closing process begins (figs. 6h-6i) and the valve is fully closed at $\mathrm{t}=2.96 \mathrm{~ms}$ (Fig. 6j) and the cycle repeats. The frequency of operation is $338 \mathrm{~Hz}$.

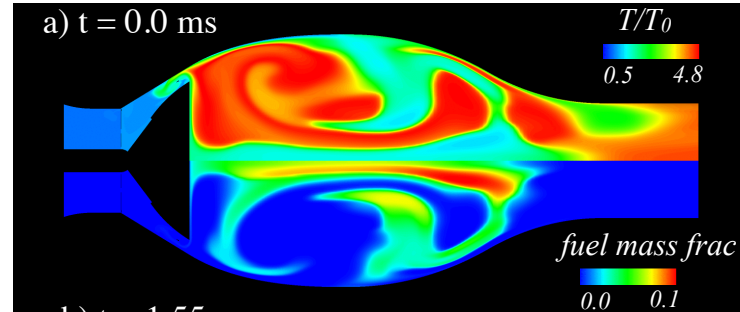

b) $\mathrm{t}=1.55 \mathrm{~ms}$

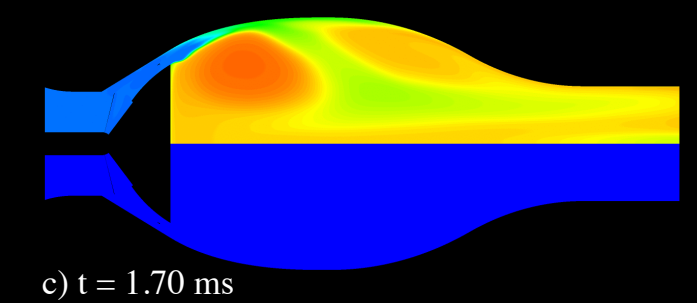

c) $\mathrm{t}=1.70 \mathrm{~ms}$

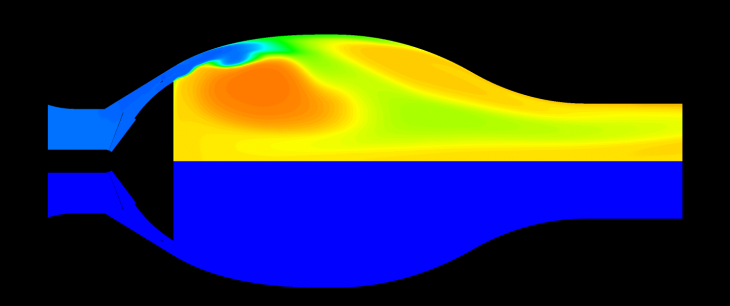

d) $\mathrm{t}=1.85 \mathrm{~ms}$

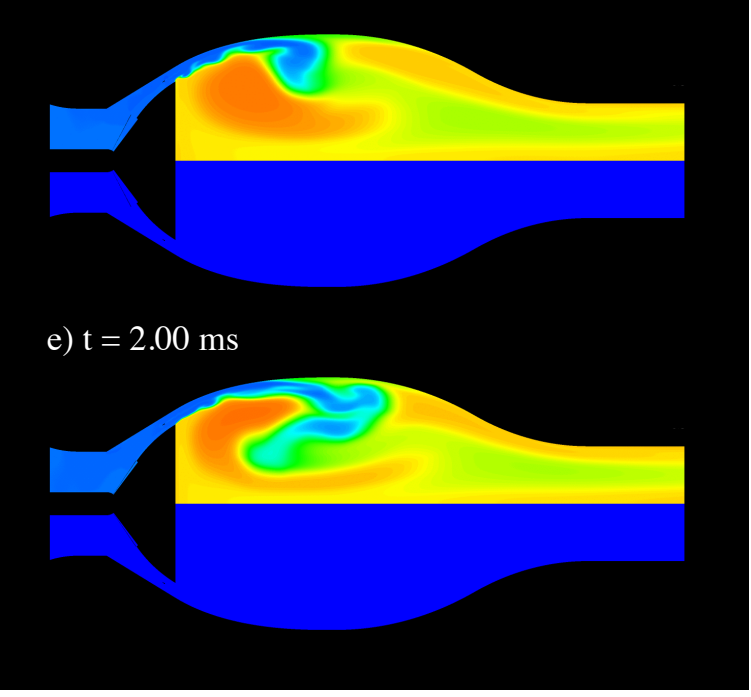

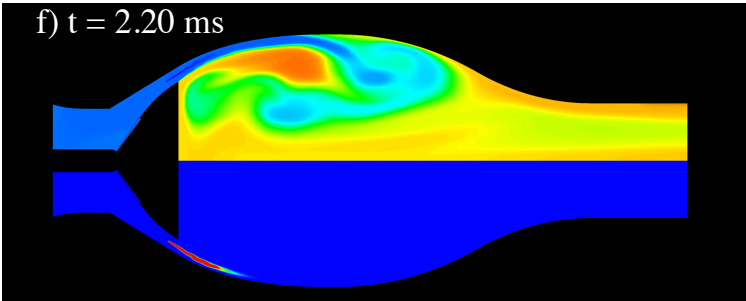

g) $\mathrm{t}=2.40 \mathrm{~ms}$

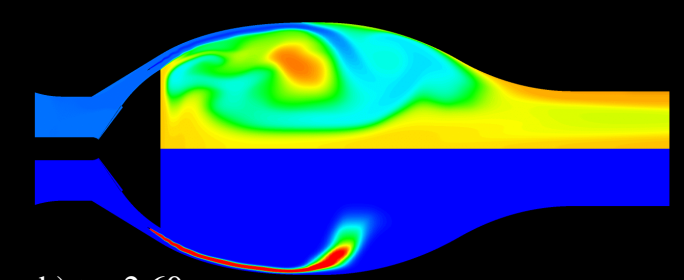

h) $\mathrm{t}=2.60 \mathrm{~ms}$

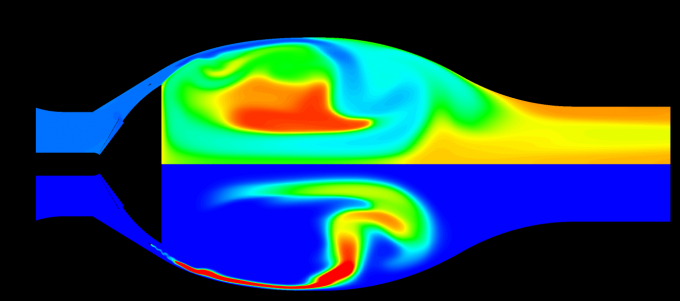

i) $\mathrm{t}=2.80 \mathrm{~ms}$
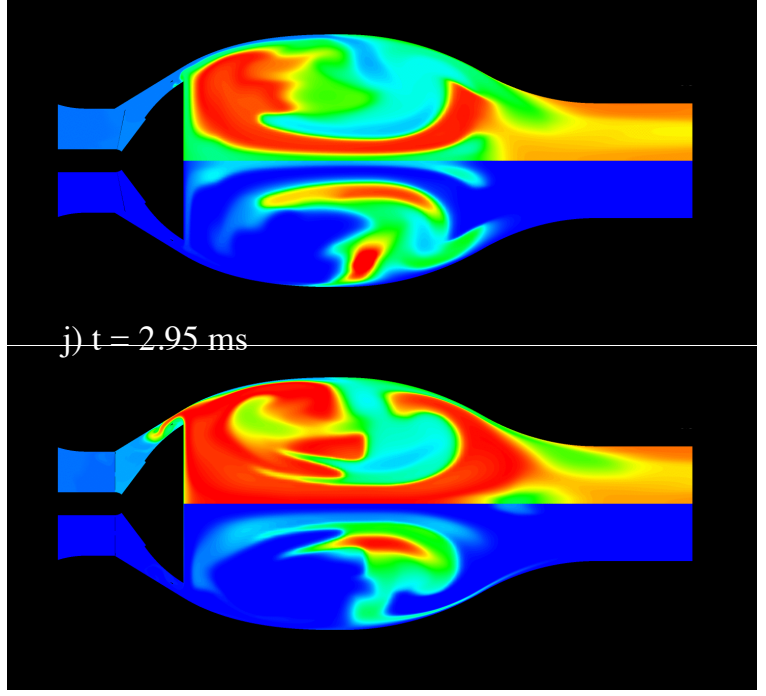

Figure 7. Temperature (top half) and fuel mass fraction (bottom half) contours during one RPC cycle. 
It is important to point out that the inlet passage should be properly designed to avoid flow separation. The configuration shown in Fig. 6 resulted in a smooth flow through the inlet without any significant flow separation. However, initial calculations with RPC inlet configurations that had a higher inlet curvature and sharper flow turning produced significant flow separation along the poppet valve surface that significantly reduced the performance of the RPC.

Figure 7 shows the combustion process in the RPC. Combustion is seen occurring mainly at the center of the primary vortex, however in Fig. 7a the flame has already propagated throughout the combustor consuming fuel also in the secondary vortex. At the $t=1.55 \mathrm{~ms}$ mark, most of the fuel has been burned (Fig. 7b). The valve begins to open at $\mathrm{t}=1.30 \mathrm{~ms}$, and air is seen entering the combustor in figs. $7 \mathrm{~b}-7 \mathrm{e}$. Fuel injection starts at $\mathrm{t}=2.15 \mathrm{~ms}$ and fuel is

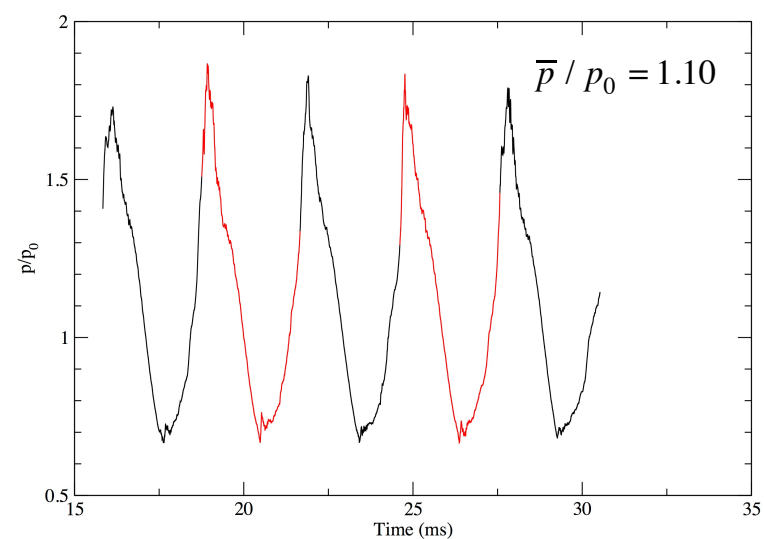

Figure 8. Combustor head-end pressure ratio. observed entering the combustor in Fig. 7f, which mixes with the incoming air and with the combustion products produced in the previous cycle. The fuel injection pressure is equal to $p_{0}$, and the duration of the fuel injection process is $0.4 \mathrm{~ms}$, resulting in an equivalence ratio of $\phi=0.77$. The results show that two counter-rotating toroidal vortices are formed in the pulse combustor, and that the fuel is split between these two vortices. Previous research ${ }^{7,8}$ has shown that maximum performance is achieved when most of the fuel is directed to the primary vortex, resulting in optimal confinement of the combustion process. Figures $7 \mathrm{~h}-7 \mathrm{j}$ show that a significant portion of the fuel ends up in the secondary vortex. RPC system optimization, along the lines carried out in Refs. 7 and 8, should be carried out in future studies to improve the fuel distribution in the poppet valve RPC configuration considered here.

The pressure history at the head-end of the combustor is shown in Fig. 8. The mean of this pressure is one of the figures of merit that characterize the performance of RPCs. ${ }^{6}$ The average combustor pressure for this case was $\bar{p} / p_{0}=1.10$, a level comparable to that obtained with the original reed valve model ${ }^{5-8}$, at corresponding equivalence ratios, which ranged from 1.11-1.14. The average mass flow rate was $\overline{\dot{m}}_{p r i}=0.23 \mathrm{~kg} / \mathrm{s}$, slightly lower than that obtained with the original reed valve configuration $(0.256 \mathrm{~kg} / \mathrm{s})$ which could partially explain the slightly lower average combustor pressure obtained in this case. It should be pointed out that the previous simulations with the original reed valve reported in Refs. 7,8 underwent significant optimization of the combustor, inlet and fuel injector components, as well as fuel injection and valve opening/closing timing. Such optimization effort has not been performed yet for the current poppet valve configuration. The fact that the performance of the non-optimized poppet valve configuration presented here is close to that of the optimized reed valve systems previously studied is very encouraging.

\section{B. Simulations of EERPCs with a CD nozzle (utilizing the original reed valve model of Refs. 5-8)}

The pulse combustor by itself is not suitable to replace a conventional combustor in a gas turbine engine. This is because the pulse combustor exhaust flow is too hot for a turbine to tolerate, and because the pulse combustor itself requires cooling similar to a conventional combustor liner. Furthermore, connecting a turbine directly downstream of the pulse combustor will completely disrupt the gasdynamic waves and their coupling with the combustion process, an interaction that is critically important for the operation of pulse combustors. ${ }^{5-8}$ Therefore, in practical

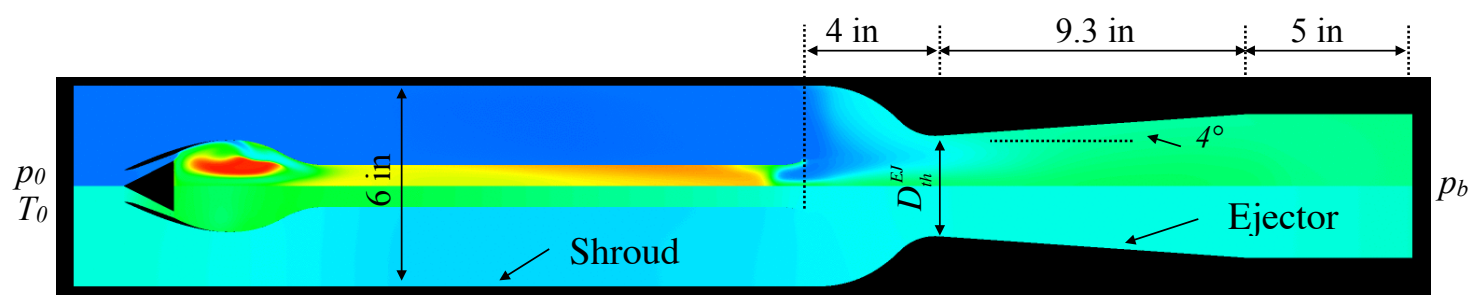

Figure 9. Temperature contours (top half) and pressure contours (bottom half) at start of cycle for the original EERPC computational model. ${ }^{7,8}$ 
applications the pulse combustor requires a shroud and some type of ejector device (i.e., an EERPC configuration). Ultimately, a can-annular arrangement of these shrouded units is envisioned to comprise a complete combustor suitable for use in a gas turbine.

In the previous studies, $, 5,7,8$ the pressure gain potential of the EERPC was investigated by applying a specified back pressure, $p_{b}$, at the EERPC exit boundary (see Fig. 9). While this approach was effective in the numerical simulations, it artificially eliminated any pressure fluctuations which could potentially occur at the exit of the EERPC. Such pressure fluctuations could have a significant impact on the performance of a turbine. Therefore, a more realistic model of the exit boundary condition occurring in a EERPC is adopted in the present study. The new model eliminates the back pressure exit boundary condition, and instead adds a CD nozzle at the exit of the EERPC to approximate more closely the effects of choked turbine inlet guide vanes that are located immediately downstream of a gas turbine combustor (see Fig. 10). The throat area of the CD nozzle can be adjusted to obtain the desired back-pressure level and total massflow rate.

Figure 10 shows results with the new EERPC computational model, based on the original RPC configuration and reed valve model of Refs. 7,8. The CD nozzle had a throat diameter, $D_{t h}^{C D}=0.86$ in for this case. As in the previous configuration, the shroud has a 6.0 in diameter and the ejector throat is located 4.0 in downstream from the end of the pulse combustor tailpipe. The ejector throat diameter was $D_{t h}^{E J}=2.4 \mathrm{in}$, which produced optimal performance in the constant $p_{b}$ model, ${ }^{7,8}$ but may not be optimal for the current configuration.
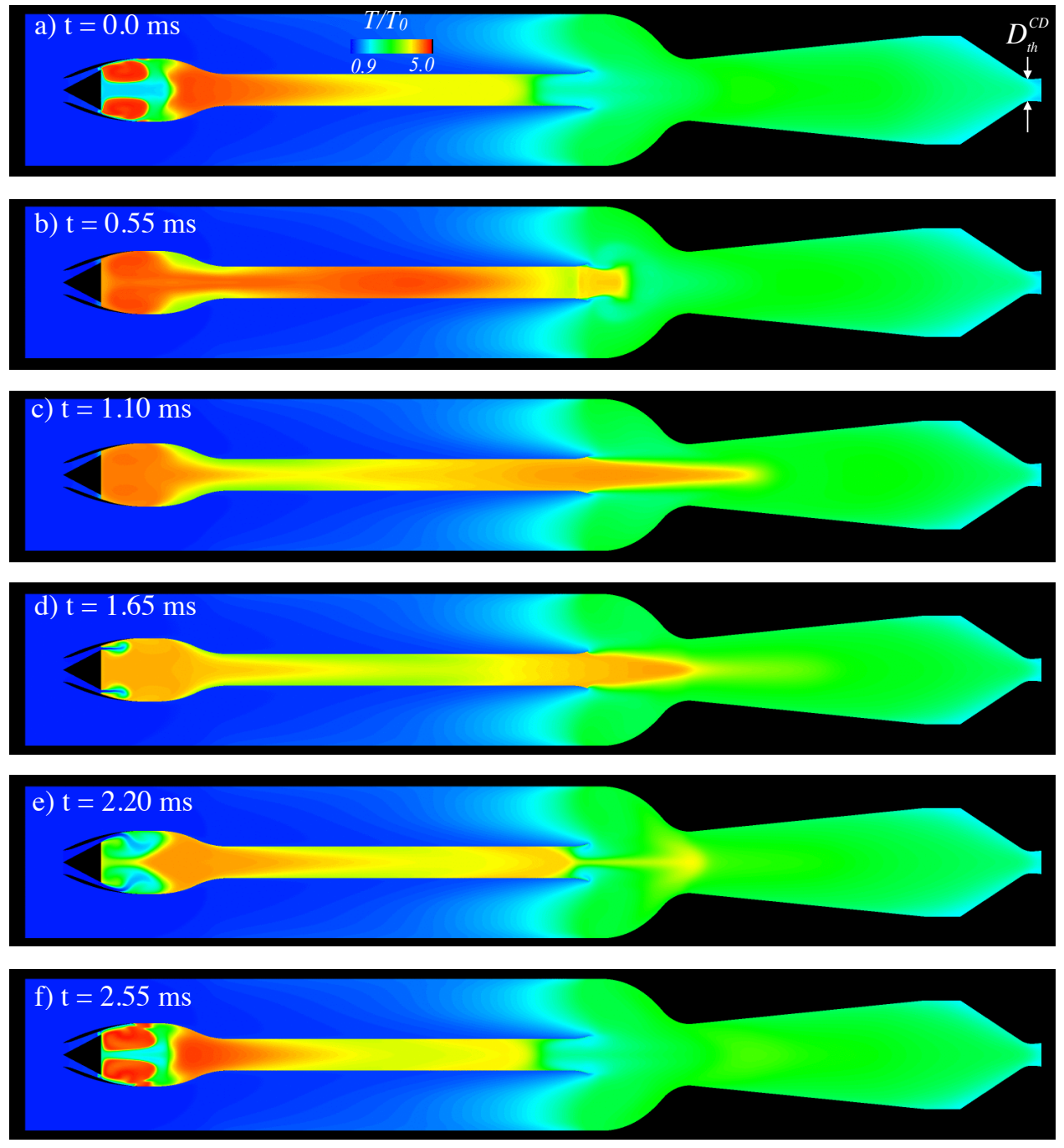

Figure 10. Temperature contours at six different times during a cycle for an EERPC with a CD exhaust nozzle. $D_{t h}^{C D}=0.86$ in. 
At the inflow plane of the computational domain, a specified total pressure and total temperature boundary condition is imposed $\left(p_{0}=10\right.$ bar and $T_{0}=550 \mathrm{~K}$ ). The air entering the EERPC is split into two streams. The primary stream flows through the pulse combustor while the secondary stream bypasses it. The two streams interact and mix in the ejector.

One of the main objectives of this study is to determine the pressure gain attainable across the shrouded EERPC without exceeding the maximum temperature allowed by a turbine. Typically, a temperature ratio of two across the combustor is encountered in aircraft gas turbine engines. Therefore, in the previous studies, a mass-averaged temperature at the exit of the shrouded EERPC was sought which is approximately two times the inlet total temperature, or $1100 \mathrm{~K}$.

The CD nozzle throat area and the pumping action of the pulse combustor determine the total airflow through the engine, and therefore will also determine the exit temperature. The system pressure gain is defined here as the difference between the mass-averaged total pressure at the exit and the inflow total pressure (expressed as a percentage of the inlet total pressure). It should be pointed out that mass-averaged total pressure has been shown to be inappropriate for pressure-gain combustion concepts with large variations in properties over the course of a cycle; however, it is convenient and acceptable in this study where variations at the ejector exit are quite small. ${ }^{6}$

Figure 10 shows temperature contours at various times during one cycle. The flow and combustion processes follow the same sequence described previously in Figs . 6 and 7 for the RPC by itself. Figure 10a shows the flowfield at the start of the cycle (Fig. 10a) followed by the blowdown process (Figs. 10b-10c), and the flowfield after the inlet valve has opened, allowing air (and fuel) to flow into the pulse combustor and forming the strong toroidal vortex system (Fig. 10d-10e). The valve is fully closed in Fig. 10f and the cycle repeats. Although the flow is unsteady, the CD nozzle remains choked throughout the entire cycle.

Figure 11 shows the pressure at the combustor head-end. The pressure history exhibits a highly uniform profile and is noticeable different from previous EERPC studies where the pressure profiles typically exhibited a perioddoubling or tripling behavior. The average combustor pressure was $\bar{p} / p_{0}=1.12$ for this case.

Figure 12 illustrates the typical process required to reach a limit cycle state in the EERPC simulations. Figure 12a shows the mass-averaged total temperature computed at the end of the diverging section of the EERPC ( $\mathrm{x}=32.6$ in) for the $D_{t h}^{C D}=0.86$ in EERPC configuration. This calculation was started from a solution previously obtained for the configuration shown in Fig. 9. After approximately 8 cycles, the mass-averaged temperature stabilized at approximately $1650 \mathrm{~K}$. Figure $12 \mathrm{~b}$ shows the mass-averaged total pressure computed at the end of the diverging section of the EERPC, and represents the system pressure gain, as defined previously. For this case, the system pressure gain converges to a value of $3.6 \%$. The frequency of operation for this cases was $371 \mathrm{~Hz}$, and the RPC equivalence ratio was $\phi=0.76$.

The mass averaged temperature at the exit of the EERPC in this case was higher than the target value $(1100 \mathrm{~K})$ due to a low bypass airflow ( $\beta=\overline{\dot{m}}_{\text {sec }} / \overline{\dot{m}}_{p r i}=0.66$ ). In order to lower this temperature the throat area of the CD nozzle needs to be increased so that more bypass air can be entrained. Therefore, calculations were carried out with a larger CD nozzle throat having a diameter of $D_{t h}^{C D}=1.02 \mathrm{in}$. The results are presented in figs. 13 and 14 . The combustor head-end pressure (Fig. 13) is not significantly affected by the modified CD nozzle. The bypass ratio increased to $\beta=1.61$, and the mass-averaged total temperature (Fig. 14a) decreased to approximately $1340 \mathrm{~K}$, closer to the target value. The frequency of operation decreased to $358 \mathrm{~Hz}$ due to the lower pressures existing at the exit of the RPC which slightly lengthens the blowdown process. The RPC equivalence ratio was unchanged. The system pressure gain for this case was approximately 3.3\%, consistent with the results obtained in previous EERPC studies. ${ }^{7,8}$ Previous analyses have shown that these levels of pressure gain should result in substantial reductions in specific fuel consumption. ${ }^{8}$

The converging portion of the $\mathrm{CD}$ nozzle could in principle reflect some of the acoustic waves back towards the RPC. The results indicate, however, that the operation or performance of the EERPC is not significantly affected by the presence of the CD nozzle.

While the mass-averaged total temperature for this case was still somewhat higher than the target goal, the calculations demonstrate that, as expected, the airflow and the exit temperature can be controlled by adjusting the throat area of the CD nozzle. The current approach to simulating EERPCs appears to be more efficient computationally than the previous back-pressure approach of Refs. 7 and 8 . The number of cycles needed to reach a limit cycle with the current configuration was between 8 and 15. The previous model required up to 25 cycles to reach a limit-cycle. ${ }^{7,8}$

The addition of an ejector and a shroud to the RPC has the additional function of reducing the temperature and velocity non-uniformities of the exhaust flow going into the turbine. This is illustrated in Fig. 15. This figure plots the centerline temperature (Fig. 15a) and velocity (Fig. 15b) as a function of time during one cycle for the 


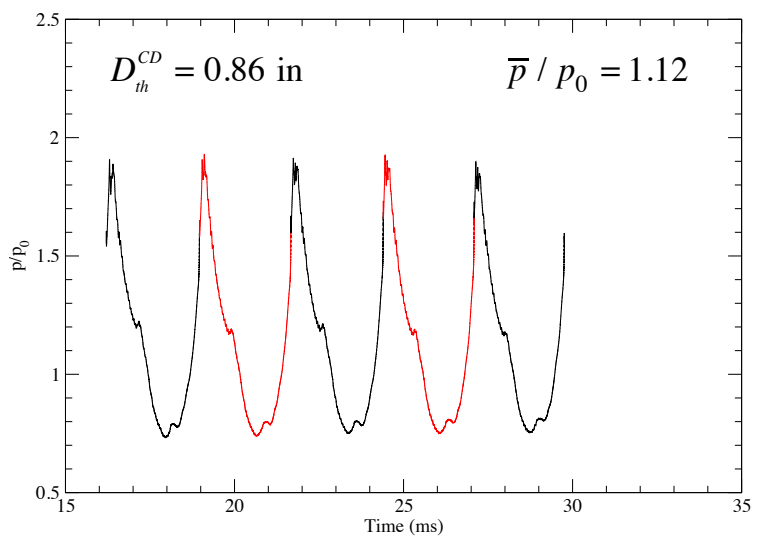

Figure 11. Combustor head-end pressure ratio for $D_{t h}^{C D}=0.86$

(a)

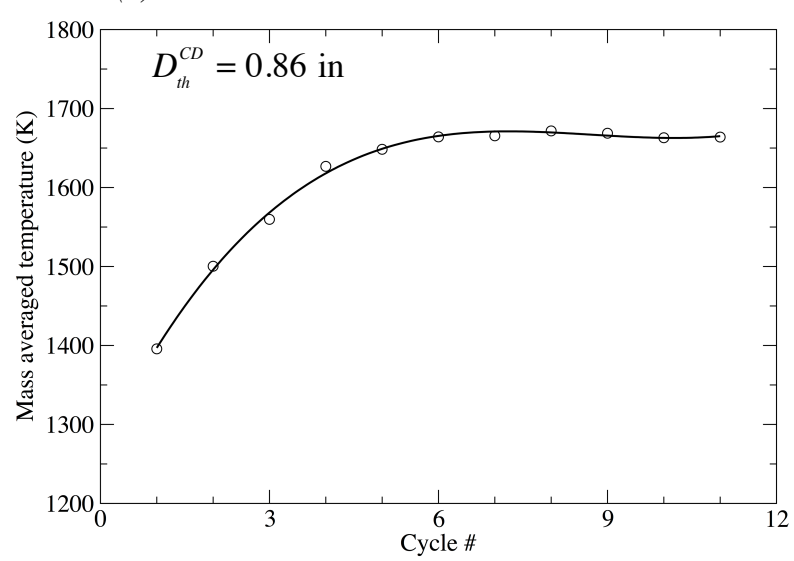

(b)

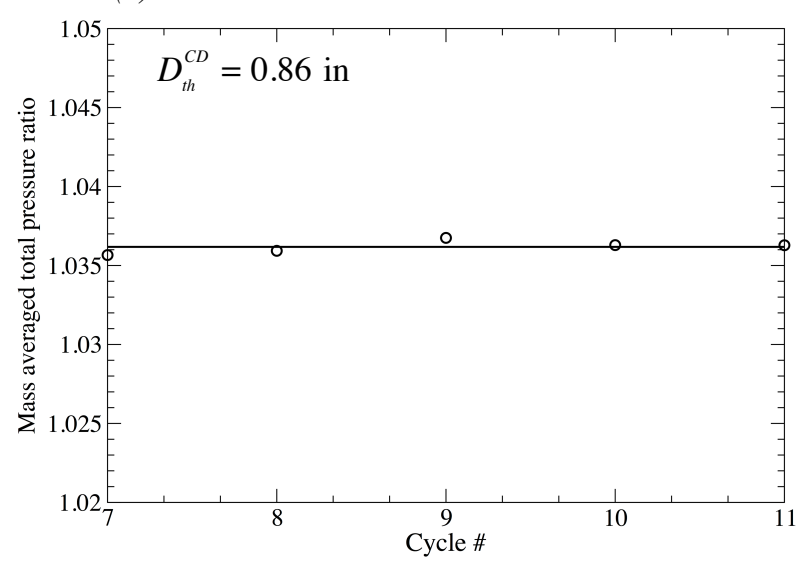

Figure 12. Mass-averaged total temperature and total pressure at the exit of the EERPC (x=32.6 in). $D_{t h}^{C D}=0.86$

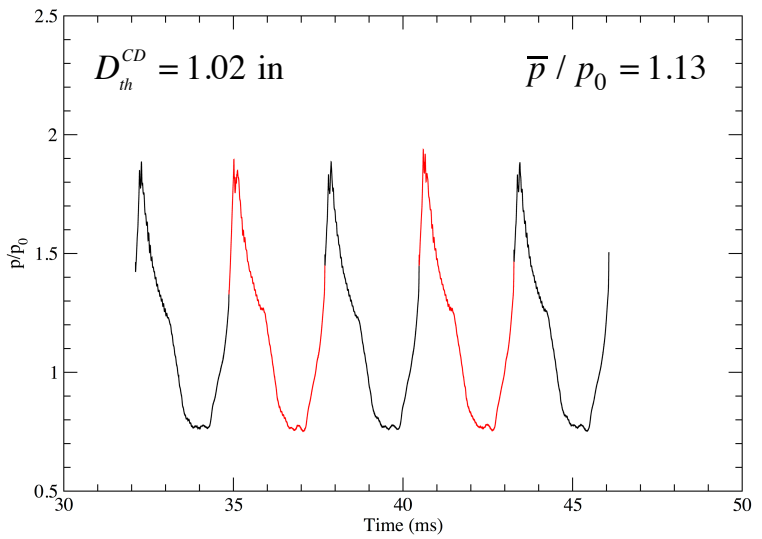

Figure 13. Combustor head-end pressure ratio for $D_{t h}^{C D}=1.02$

(a)
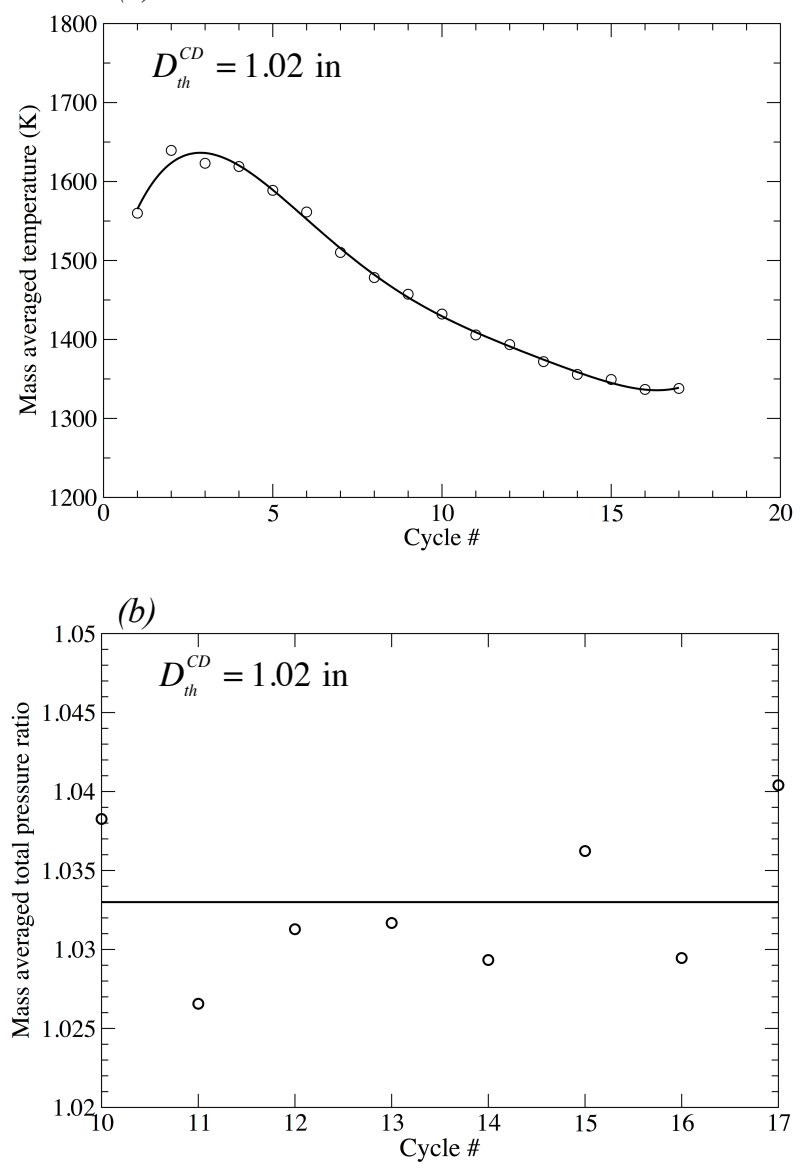

Figure 14. Mass-averaged total temperature and total pressure at the exit of the EERPC (x=32.6 in). $D_{t h}^{C D}=1.02$ 

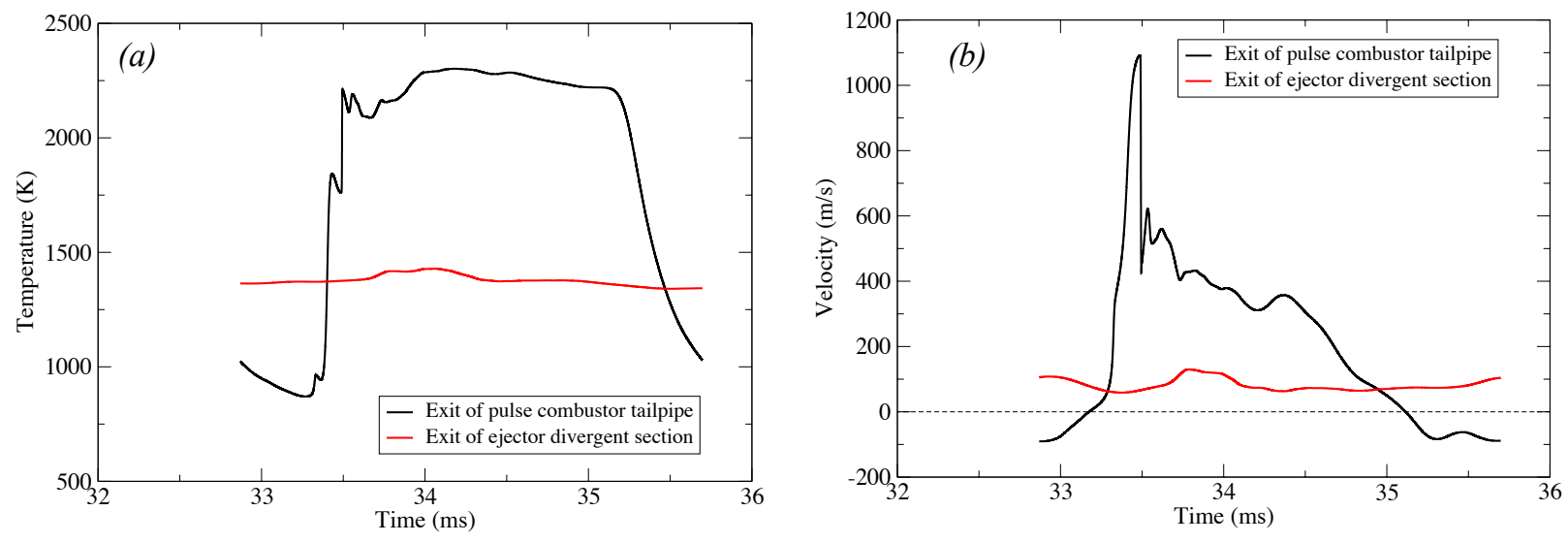

Figure 15. Centerline temperature (a) and axial velocity (b) at the EERPC exit plane ( $x=32.6$ in).

$D_{t h}^{C D}=1.02$ in EERPC configuration. Quantities are plotted at the exit of the pulse combustor $(\mathrm{x}=19.3 \mathrm{in})$ and at the end of the diverging portion of the ejector $(\mathrm{x}=32.6 \mathrm{in})$. The average temperature at the exit of the pulse combustor was $\bar{T}_{p c}=1841 \mathrm{~K}$, with a standard deviation of $\sigma_{p c}^{T}=545 \mathrm{~K}$. In comparison, the average temperature at the end of the diverging portion of the ejector was $\bar{T}_{e j}=1377 \mathrm{~K}$ with a standard deviation of $\sigma_{e j}^{T}=24 \mathrm{~K}$. The average velocity at the exit of the pulse combustor was $\bar{U}_{p c}=198 \mathrm{~m} / \mathrm{s}$, with a standard deviation of $\sigma_{p c}^{U}=254 \mathrm{~m} / \mathrm{s}$, while the average velocity at the end of the diverging portion of the ejector was $\bar{U}_{e j}=82 \mathrm{~m} / \mathrm{s}$, with a standard deviation of $\sigma_{e j}^{U}=19 \mathrm{~m} /$ s.

The computational model used in this study allows for the calculation of the pressure fluctuations at the exit of the EERPC. For the $D_{t h}^{C D}=1.02$ in EERPC configuration, the centerline RMS pressure fluctuations were less than $7 \%$ of the mean pressure. It is clear that both goals of reducing the average exhaust temperature and the level of flow non-uniformities have been accomplished with the EERPC configuration.

\section{Conclusions}

This study focused on two enhancements to previous RPC models, related to the valve and ejector components. In the first part of the study, a new inlet poppet valve system was investigated, replacing the reed valve used previously, which tends to have a short life due to the high temperatures and impact forces experienced during operation, and also due to the fact that it has to be flexible and therefore thin. The poppet valve would be actuated in a similar way as those found in internal combustion engines, and should significantly increase the robustness and reliability of the system (although at the cost of added mechanical complexity).

An approximate poppet valve model was developed and analyzed. The results indicated that the new valve/inlet configuration can achieve pulse combustor average pressure levels comparable to those attained with the original RPC configuration. However, system optimization, along the lines carried out for the reed valve based RPC configurations, should be performed in order to determine the maximum system performance.

Engineering development of a poppet valve system for a RPC is currently under way at the NASA Glenn Research Center with the goal of replacing the current reed valve based RPC experimental device.

The second part of the study examined a new computational approach to approximate the exit boundary effects of turbine inlet guide vanes present immediately downstream of an EERPC. Instead of specifying a back pressure at the EERPC exit boundary (as was done in previous studies) the new model added a CD nozzle at the exit of the EERPC.

The results indicated that the addition of the CD nozzle is an effective method to approximate the exit boundary conditions imposed by the presence of a choked turbine. The results demonstrated that the total mass flow rate and mass averaged temperature at the exit of the EERPC can be controlled by adjusting the throat area of the CD nozzle. System pressure gain levels of up to $3.6 \%$ were obtained, although this was accompanied with a mass averaged temperature higher than the target value $(1600 \mathrm{~K}$ vs $1100 \mathrm{~K})$. At lower mass averaged exit temperature 
$(1340 \mathrm{~K})$ the system pressure gain was approximately $3.3 \%$, consistent with previous studies. Previous studies have shown that these levels of pressure gain should result in substantial reductions in specific fuel consumption.

The more acoustically reflective boundary imposed by a CD nozzle placed at the end of the EERPC, as compared to a constant pressure surface, does not appear to significantly affect operation or performance.

This research effort, carried out over the past few years, has led to an EERPC concept that, in principle, can replace a conventional combustor in a gas turbine engine. The EERPC concept that has emerged, can operate efficiently at high pressure conditions, and produce system pressure gains above $3 \%$, with a reasonable expectation of structural robustness and reliability, and with a turbine compatible outflow. In addition, the EERPC has the potential to reduce NOx emissions to levels below those produced by conventional gas turbine engines.

\section{Acknowledgments}

This study was supported by NASA under contract number NNC13TA84T.04. The computational resources were provided by the NASA Advanced Supercomputing (NAS) Division.

\section{References}

1. Lefebvre, Arthur, H., Gas Turbine Combustion, Taylor and Francis, 1999, Chapt. 4.

2. Jones, S.M. and Paxson, D.E., "Potential Benefits to Commercial Propulsion Systems from Pressure Gain Combustion," AIAA paper 2013-3623, July 2013.

3. Paxson, D.E. and Dougherty, K., "Ejector Enhanced Pulsejet Based Pressure Gain Combustors: An Old Idea With a New Twist," AIAA paper 2005-4216, July 2005.

4. Keller, J.O. and Bramlette, T.T., "Pulse Combustion: The Importance of Characteristic Times," Combustion and Flame, 75, 33-44 (1989).

5. Yungster, S., Paxson, D.E. and Perkins, H.D.,"Computational Study of Pulsejet-Driven Pressure Gain Combustors at High-Pressure," AIAA paper 2013-3709, July 2013.

6. Yungster, S., Paxson, D.E. and Perkins, H.D.,","Effect of Fuel Injection and Mixing Characteristics on Pulse-Combustor Performance at High-Pressure," AIAA paper 2014-3728, July 2014.

7. Yungster, S., Paxson, D.E. and Perkins, H.D.,"Parametric Study of Pulse combustor-Driven Ejectors at High-Pressure," AIAA 2015-4190, July 2015.

8. Yungster, S., Paxson, D.E. and Perkins, H.D.,'Numerical Investigation of Shrouded Ejector-Enhanced Pulse Combustor Performance at High-Pressure," To be published in Journal of Propulsion and Power.

9. Yungster, S. and Radhakrishnan, K., A Fully Implicit Time Accurate Method for Hypersonic Combustion: Application to Shock-Induced Combustion Instability. Shock Waves, 5, 293-303, (1996).

10. Yungster, S. and Radhakrishnan, K., Pulsating one-dimensional detonations in hydrogen-air mixtures. Combustion Theory and Modeling, 8, 745-770, (2004).

11. Spalart, P. R. and Allmaras, S. R., A One-Equation Turbulence Model for Aerodynamic Flows, La Recherche Aerospatiale, 1, 5-21, (1994).

12. Ajmani, K., Kundu, K. and Penko, P.F., "A Study on Detonation of Jet-A Using a Reduced Mechanism," AIAA paper 2010-1515, January 2010. 